\title{
Biomarkers, type II collagen, glucosamine and chondroitin sulfate in osteoarthritis follow-up: the "Magenta osteoarthritis study"
}

\author{
M. Scarpellini $\cdot$ A. Lurati $\cdot$ G. Vignati $\cdot$ \\ M. G. Marrazza $\cdot$ F. Telese $\cdot$ K. Re $\cdot$ A. Bellistri
}

Received: 2 March 2008/ Accepted: 7 April 2008/Published online: 28 May 2008

(C) Springer-Verlag 2008

\begin{abstract}
Background The purpose of the present study was to determine relationship between disease activity, systemic markers of cartilage degradation, urinary C-terminal crosslinking telopeptides of type II collagen (uCTX-II), and bone degradation, urinary C-terminal cross-linking telopeptides of type I collagen (uCTX-I), structural progression of osteoarthritis (OA) and potential therapeutic efficacy of type II collagen (COLLII) in combination with glucosamine and chondroitin sulfate (GC).

Materials and methods An observational retrospective study, 1-year follow-up, on 104 patients with OA (nodular osteoarthritis of the hand, erosive osteoarthritis of the hand, EOA, osteoarthritis of the knee or hip) who were treated with GC or glucosamine, chondroitin sulfate and collagen type II (GCC). The following information was collected at entry: demographics, BMI, characteristics of OA, patient global assessment (VAS), C-terminal cross-linking telopeptides of collagen types I (uCTX-I) and II (uCTX-II) and radiographs. After 6 months: VAS, uCTX-I and uCTXII. After 1 year: VAS, uCTX-I, uCTX-II and radiographs. Results After 6 months and 1 year of treatment VAS, uCTX-I and uCTX-II mean values were significantly lower than the baseline. 57 were treated with GCC and 47 with GC. The group that received GCC showed a similar VAS mean value after 6 months and 1 year when compared with
\end{abstract}

M. Scarpellini ( $₫)$ · A. Lurati · M. G. Marrazza · F. Telese . K. Re $\cdot$ A. Bellistri

Rheumatology Unit, Magenta Hospital Italy,

Via al Donatore di Sangue 50, 20013 Magenta, Milan, Italy

e-mail: magda.scarpellini@ao-legnano.it

G. Vignati

Endocrine and Metabolic Disease Center, Magenta Hospital Italy, Via al Donatore di Sangue 50, 20013 Magenta, Milan, Italy the group treated with GC. uCTX-I and uCTX-II mean level was lower in the group treated with GCC $(P<0.05)$. Radiological score (Kellgren and Lawrence summarized score for hands) after 1 year showed a reduced progression compared to the baseline in the hand osteoarthritis group, especially after GCC treatment $(P<0.05)$. Finally, uCTX-I has better correlation with radiological score and with GC in the EOA subgroup (Pearson index: $R=0.44$ ).

Conclusions (a) UCTX-I and UCTX-II proved to be useful biomarkers in OA monitoring; (b) UCTX-I is better correlated with hand EOA and could represent a potential further marker to assess the evolution of EOA bone damage; (c) GC slow down OA progression; (d) finally COLLII could represent a further protective factor in OA cartilage.

Keywords Osteoarthritis - Type II collagen · Cartilage · uCTX-I · uCTX-II

\section{Introduction}

Articular cartilage is constructed with hyaline cartilage tissue. It is composed of chondrocytes located in lacunae and in the extracellular matrix. The chondral matrix contains water, collagen, proteoglycans, non-collagenous matrix proteins and lipids. Articular cartilage is divided into four zones-superficial, intermediate, deep and calcified-according to morphology, the orientation of collagen fiber, and the proteoglycan content. The dominant collagen of this tissue is type II collagen (COLLII), together with smaller quantities of other collagens (IX, XII). Numerous studies have shown that chondrocytes also have tissuespecific antigens, which induce the introduction of antibodies in patients with cartilage grafts, as well as those with osteoarthritis (OA) [1]. 
Moreover, it has been demonstrated that some chondrocytes can have migratory capacity, and the migratory ones can synthesize COLLII but not type I collagen (COLLI) [2]; this can be interpreted as further evidence that joint damage involves mostly COLLII.

Collagens are a big family of proteins, the main one forming connective tissue in all higher animals. Connective tissues contain a mixture of cells, proteins, complex polysaccharides and inorganic constituents. COLLII, like elastine and proteoglycans, is located in the extracellular matrix and is produced by fibroblasts.

The functional property of COLLII is to give strength and flexibility to the connective tissue, resisting the tensions suffered in the direction of its fibers. At the moment there are 28 different identified types of collagen. In connective tissue, native COLLII is arranged in fibrils. Its function consists in giving strength and flexibility to the connective tissue, resisting the tensions suffered in the direction of its fibers. Also, COLLII is present in joints. It has a special configuration that gives particular elastic properties to protein: collagen fibers are located in the extracellular matrix and have the capacity to increase or reduce their volume according to the degree of compression to which they are subjected. Therefore it protects the organs and tissues from rupture and loss of form or structure when they are stressed by sudden or violent movements. Besides its structural role in tissues and organs, collagen is also important for the development of tissues, one of its functions is to influence the differentiation and proliferation of non-specialized cells. Over the last years numerous molecular markers of cartilage breakdown have been used and evaluated to predict the structural progression of OA. Among the used markers, one of the most reliable resulted urinary C-terminal cross-linking telopeptides of type II collagen (uCTX-II) [3-11]. COLLI is a metalloprotease cleavage product of human articular cartilage and is normally used to test osteoclastic activity and to quantify bone reabsorption [10]. The article aims at providing a comparison between two associations (glucosamine-chondroitin sulfate and glucosamine-chondroitin sulfate-native COLLII partially hydrolyzed) in a cohort of Italian patients with OA. Furthermore, the role of urinary C-terminal cross-linking telopeptides of type I collagen (uCTX-I) and uCTX-II as biomarkers has been evaluated.

\section{Materials and methods}

Patients and methods

This was an observational retrospective 1-year follow-up study. This study has considered patients who came to our hospital between October 2006 and October 2007 and who were affected by osteoarthritis of the hand, hip or knee (fulfilling the American College of Rheumatology Criteria) [12].

At baseline the following characteristics were collected: age, gender, BMI. Each patient was evaluated at baseline, after 6 months and after 1 year with a general rheumatologic assessment, a patient global assessment $(0-100 \mathrm{~mm}$ VAS), uCTX-I and uCTX-II measurement.

For the urinary evaluation of degradation products of C-terminal telopeptides of Type I human collagen the Urine CrossLaps ${ }^{\circledR}$ ELISA Kit has been employed and for products of C-terminal telopeptides of type II the Urine CartiLaps ${ }^{\circledR}$ ELISA kit (both from Nordic Bioscience Diagnostic-DK, Italian subsidiary Pantec-Turin) has been employed. The method gives a quantitative determination employing a competitive enzyme immunosorbent assay on micro-titer wells by using urine from second morning void, the concentration is referred to the creatinine excretion with results as nanogram $/ \mathrm{mmol}$ of creatinine.

At baseline and after 1 year a radiological evaluation of hands, hip or knee as needed was observed. All radiographs were evaluated by two experienced rheumatologists and the Kellgren and Lawrence score was used [13]. Obviously, patients whose required parameters were (partially) absent from our archives were not considered. Secondary OA, due for example to fracture, inflammatory diseases (such as rheumatoid arthritis) or Paget disease, was an exclusion criterion. Patients with current corticosteroid therapy and/ or anti-osteoporotic drugs and/or renal or hepatic dysfunction in the year before onset of the study were excluded too.

Hundred and four patients were thus included in the study (95 females, 9 males), mean age was $61.4 \pm 7.2$ years. Thirty patients were affected by hand EOA, 54 by hand OA and 20 distributed between knee or hip OA.

Forty-seven were treated with glucosamine $1,000 \mathrm{mg}+$ chondroitin sulfate $1,000 \mathrm{mg}(\mathrm{GC})$ and 57 with glucosamine $1,000 \mathrm{mg}+$ chondroitin sulfate $1,000 \mathrm{mg}+$ native COLLII partially hydrolyzed $2 \mathrm{mg}$ (GCC).

The study was performed in accordance to the Declaration of Helsinki.

Statistical analysis

Continuous variables were analyzed in terms of mean \pm standard deviation.

Standard Student's $t$-test for paired samples or one-way ANOVA were performed for comparing data, as needed. A $P$-value $<0.05$ has been valued as significant.

All analyses were carried out with SPSS software for windows Ver 13.0. 


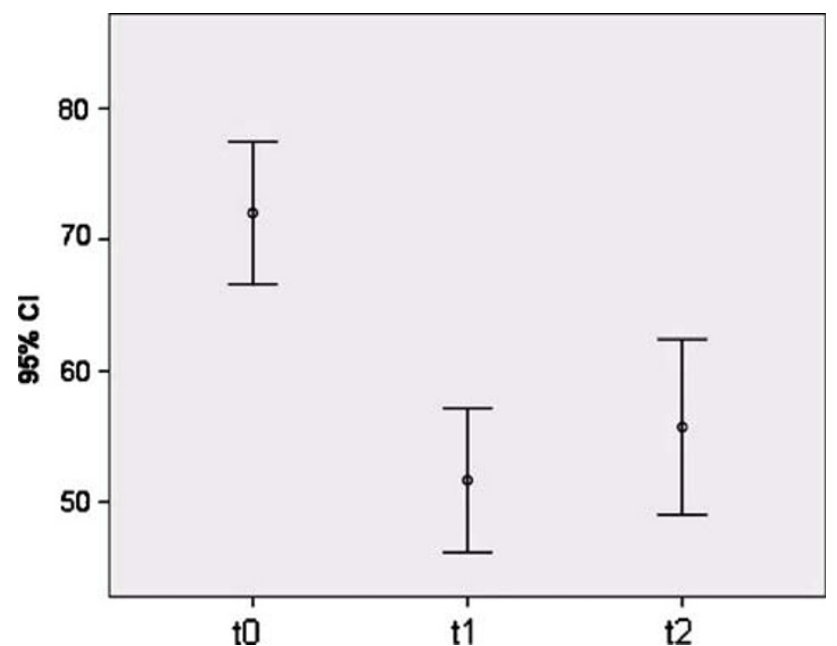

Fig. 1 VAS in the whole population $(\mathrm{GC}+\mathrm{GCC})$

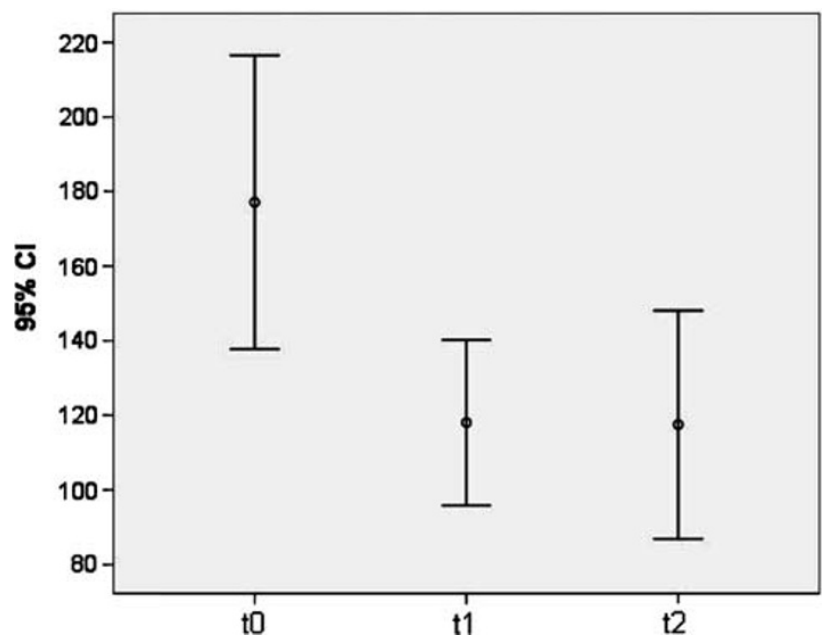

Fig. 2 Urinary C-terminal cross-linking telopeptides of type I collagen in whole population $(\mathrm{GCC}+\mathrm{GC})$

\section{Results}

Analyzing the whole population, VAS (Fig. 1) was reduced significantly after 6 months (t1: $P=0.014)$, and was still low after 1 year (t2: $P=0.004)$ compared to the baseline (t0) in both groups (GC and GCC). The same is true for uCTX-1 (Fig. 2), t1: $P=0.001$ and t2: $P=0.002$. There was no significant difference between GC and GCC. uCTX-I in hand OA and hand EOA is more reduced in the group treated with GCC at $\mathrm{t} 2(P=0.026)$ compared to the other group (Fig. 3).

Urinary C-terminal cross-linking telopeptides of type I collagen in the EOA subgroup is more significantly reduced $(P=0.017)$ at $\mathrm{t} 2$ with $\mathrm{GCC}$ compared to GC (Fig. 4).

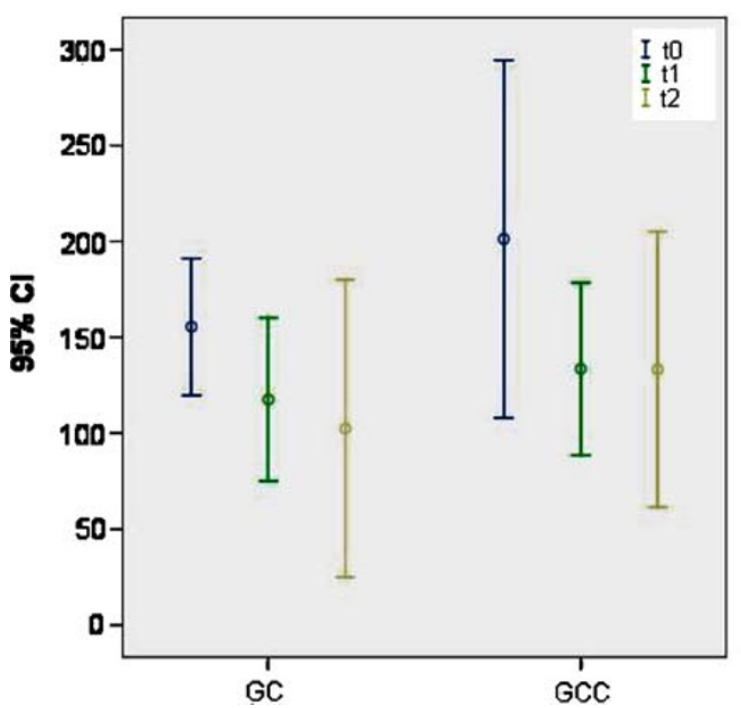

Fig. 3 Urinary C-terminal cross-linking telopeptides of type I collagen in hand OA and hand EOA, GCC vs. GC

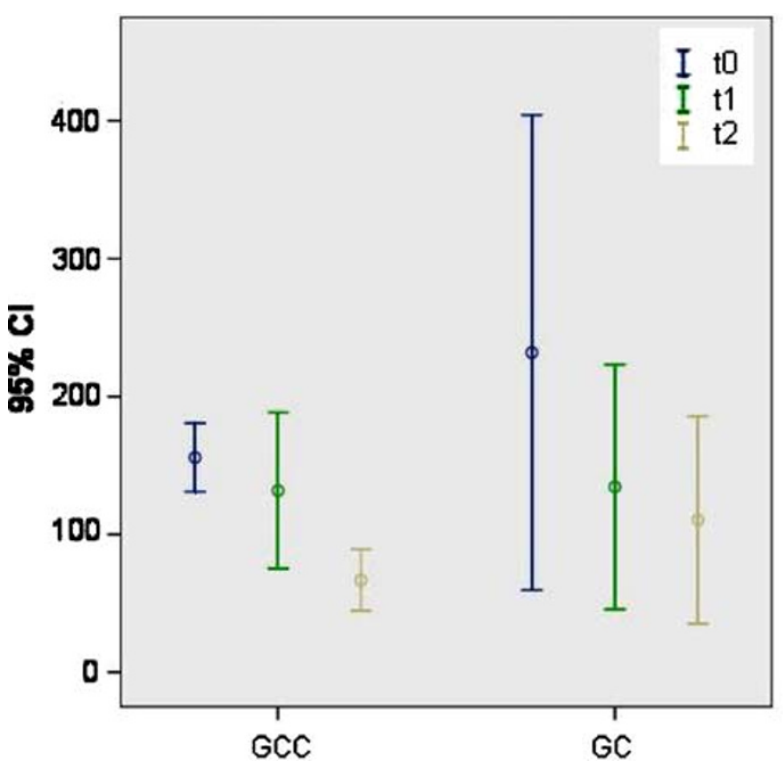

Fig. 4 Urinary C-terminal cross-linking telopeptides of type I collagen in EOA group, GCC vs. GC

Urinary C-terminal cross-linking telopeptides of type I collagen has a higher baseline in the EOA subgroup.

Urinary C-terminal cross-linking telopeptides of type II collagen is significantly reduced in the whole population both at $\mathrm{t} 1(P=0.003)$ and $\mathrm{t} 2(P=0.002)$ and with both treatments (Fig. 5); patients treated with GCC improve more quickly and steadily over 1 year, whereas patients treated with GC show smaller improvements which tend to regress at $\mathrm{t} 2$.

To make a more accurate comparison of the uCTX-II improvements, we chose the group affected by hand 


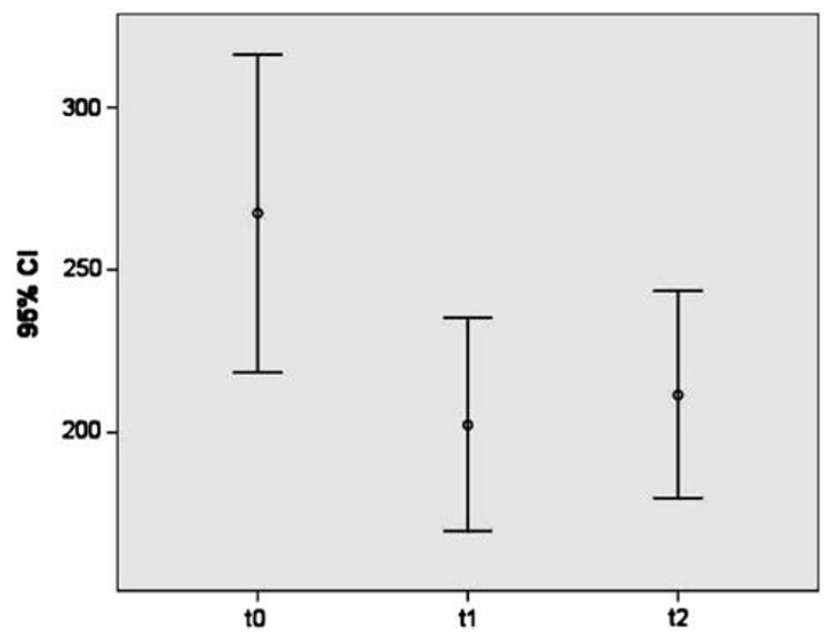

Fig. 5 Urinary C-terminal cross-linking telopeptides of type II collagen in whole population $(\mathrm{GCC}+\mathrm{GC})$

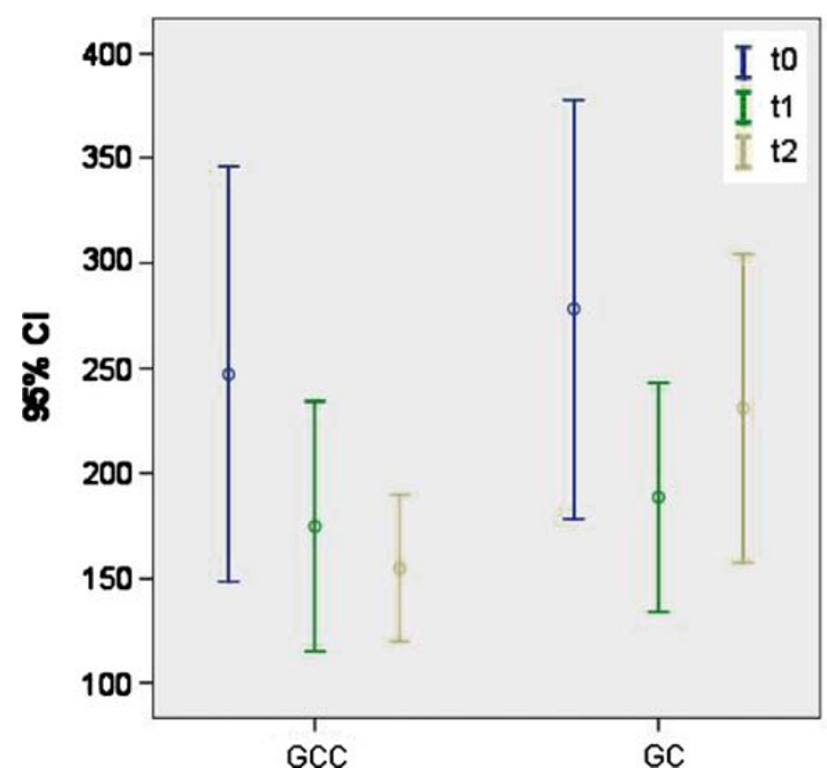

Fig. 6 Urinary C-terminal cross-linking telopeptides of type II collagen in hand OA and hand EOA, GCC vs. GC

arthritis $(\mathrm{OA}+\mathrm{EOA})$ because it is the more numerous and homogeneous from many points of view (sex, age, BMI). uCTX-II is significantly reduced at $\mathrm{t} 1(P=0.01)$ already, with both treatments. However, at $2(P=0.017)$ patients treated with GCC show further improvements whereas patients treated with GC tend to regress (Fig. 6).

From the radiological point of view, in the hand arthritis group $(\mathrm{OA}+\mathrm{EOA})$, patients with GCC show a decrease in the bone decay rate over 1 year $(P=0.009)$ (Fig. 7), starting from similar radiologic score at t0. Figure 8 is like Fig. 7, but limited to patients with hand-EOA $(P=0.018)$, starting with similar radiologic score at $\mathrm{t} 0$, as well.

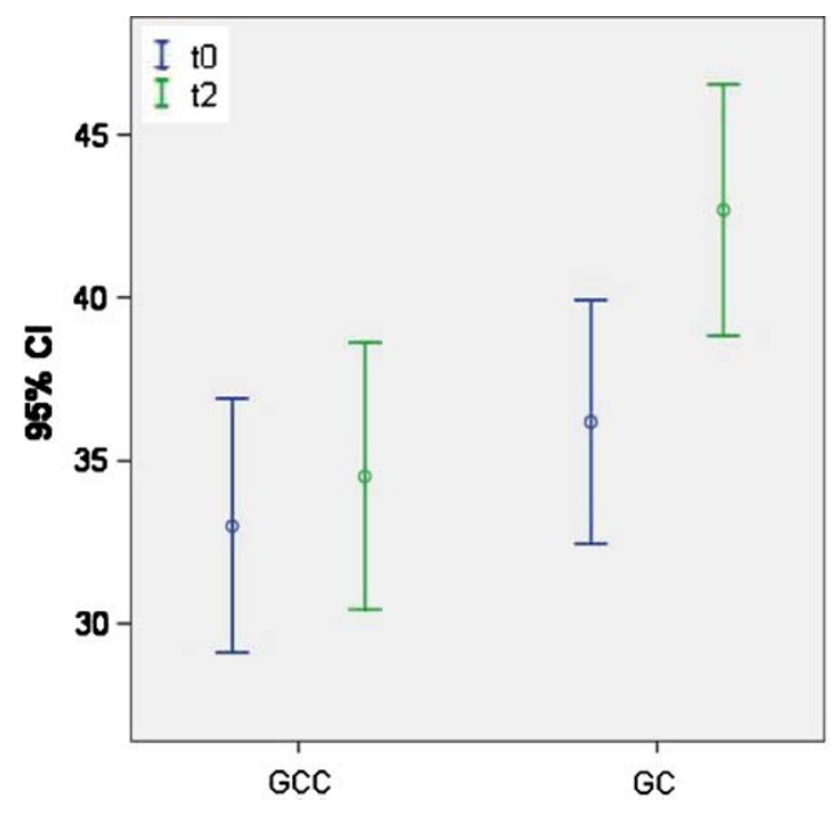

Fig. 7 Evolution of radiological score in hand OA and hand EOA, GC vs. GCC

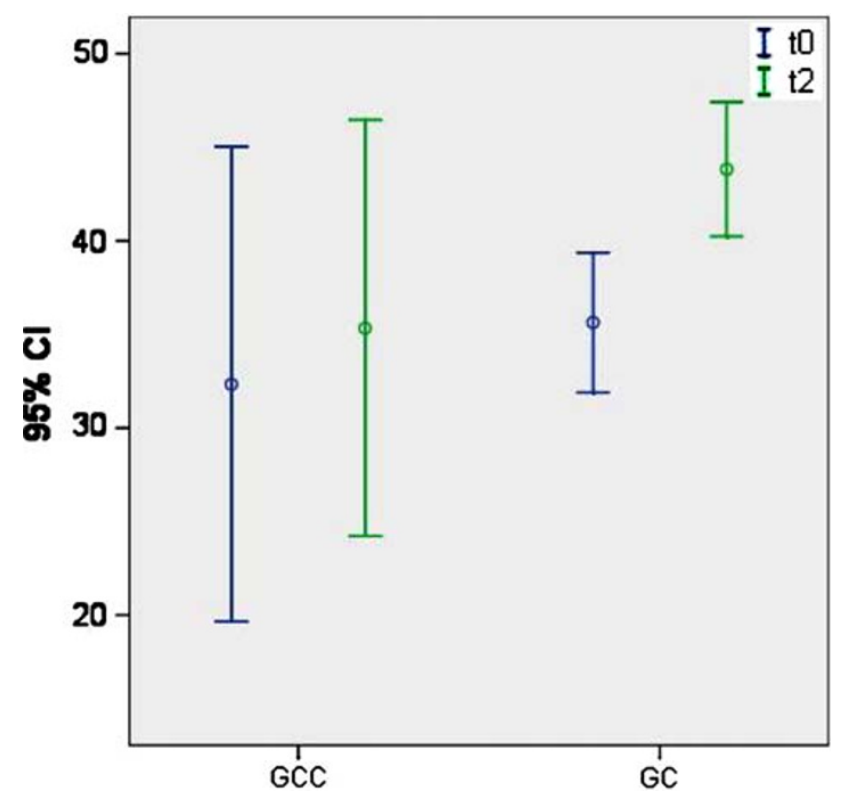

Fig. 8 Evolution of radiological score in hand EOA, GC vs. GCC

In EOA patients, analyzing the possible correlation between radiological data, uCTX-I and uCTX-II, we found a significant correlation between radiological score (at t2) and UCTX-I (both at $\mathrm{t} 1$ and $\mathrm{t} 2$ ), with a Pearson index $R=0.59, P=0.0008$.

There is no correlation between radiological data and uCTX-II.

In hand OA patients, there is a weak correlation between radiological data and $\mathrm{uCTX}-\mathrm{II}$, with a Pearson index 


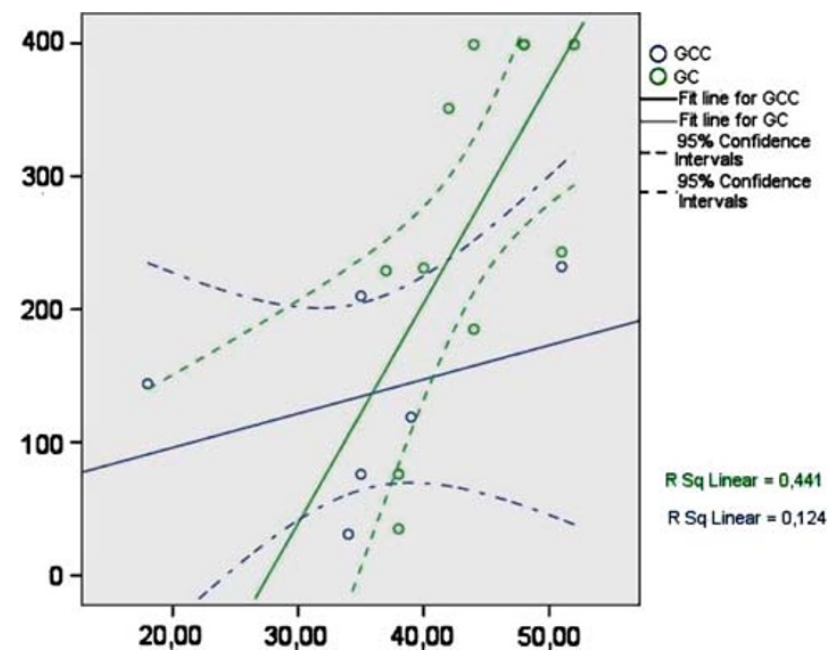

Fig. 9 Correlation between radiological score (abscissa) and uCTX-I (ordinate) in hand EOA, GC vs. GCC

$R=0.315, P=0.045$. This datum seems to confirm that the EOA group is better described by uCTX-I than uCTXII.

Pearson index between radiological score and uCTX-I in the EOA group treated with $\mathrm{GC}$ is $R=0.44$. Pearson index between radiological score and UCTX-I in the EOA group treated with GCC is $R=0.12$. This datum is consistent with the fact that GCC slows down the rate of increase of radiological score, thus UCTX-I is a better indicator of radiological evolution in the GC group compared to the GCC group, where the effect of the drug weakens the correlation between these two variables (Fig. 9).

\section{Discussion}

$\mathrm{OA}$ is the most common and frequent of the rheumatic diseases. Research on pathogenesis has suggested new ideas both in new diagnostic tests and in therapy.

Our study, in accordance with many AA, has confirmed the validity of uCTX-II in the diagnosis and quantitative analysis of cartilage breakdown $[10,11]$ also during follow up, independently of area, localization and histological grading of affected joints [14] and atrophic or hypertrophic patterns of OA [7].

In our study, also uCTX-I has demonstrated itself interesting. Recently, as a matter of fact, the presence of bone resorption has been recognized as a risk factor for OA progression. A recent study [15] demonstrated that general bone resorption, indicated by serum biomarker measurements, is increased in patients with progressive knee OA. These results confirmed the role of bone changes in the pathogenesis of OA. It is generally believed that degeneration of cartilage in $\mathrm{OA}$ is characterized by two phases: a biosynthetic phase during which the cells resident in cartilage, the chondrocytes, attempt to repair the damaged extracellular matrix; and a degradation phase, in which the activity of enzymes produced by the chondrocytes digests the matrix, matrix synthesis is inhibited, and the consequent erosion of the cartilage is accelerated. The cellular reaction pattern during the osteoarthritic disease process is at first glance rather heterogeneous. However, the reaction patterns can basically be summarized in five categories: (a) proliferation and cell death (apoptosis), (b) changes in synthetic activity, (c) changes in degradation, (d) phenotypic modulation of the articular chondrocytes, and (e) formation of osteophyte. Several factors such as retinoic acid, bromodeoxyuridine and IL-1, induce so-called "dedifferentiation" or modulation of the chondrocytes phenotype to a fibroblast-like phenotype. The chondrocytes stop expressing aggrecan and collagen type II, though they are still very active cells and express collagen types I, III and V [16]. Some AA [17] have reviewed the evidence that $\mathrm{OA}$ abnormal osteoblasts are responsible: (a) to maintain the abnormal mineralization, (b) to release factors that can modify both osteoblasts and chondrocytes functions and (c) to degrade articular cartilage. OA osteoblasts release more IL-6, CXCL12, CXCL13 and leptin that can also directly modulate COLLI synthesis, promote articular cartilage degradation and contribute to inflammatory state observed in OA. Production of an abnormal collagen matrix and a soluble factor(s) by OA osteoblasts leads to an abnormal osteoid matrix not mineralizing normally. This putative factor(s) contributes to cartilage degradation but also to abnormal osteoblasts cell function, measurable also as uCTX-I. Marcelli et al. [18] underline the relationship between hand OA and bone mineral density and Zoli et al. [19] confirm it also in hand EOA, a destructive form of primary OA. The most important problem for therapy is the large pathogenetic process number. Obviously therapeutic intervention in order to control both joint cartilage collagen content and bone content have to be considered in OA management. Numerous pharmaceutical and nutriceutical agents [20] have been developed and are used also in combination, to improve the efficacy in delaying the progression of structural changes in OA cartilage $[21,22]$. Actually many AA have described the efficacy and potential disease-modifying effect of glucosamine, chondroitin sulfate, avocado soybean unsaponifiables, diacerhein, intraarticular hyaluronan, ginger, doxycycline, ascorbic acid, manganese, growth factors [23].

Our group wanted to test the potential capacity of native COLLII, partially hydrolyzed, in association with GC, to lessen the damage and progression of OA, based on the above. Collagen gives bone its flexibility, helping it to 
resist tension. COLLII helps to promote new cartilage synthesis and reduces oxidative damage to the joints. In our study a synergic mix of natural compounds, as GCC, worked together to reduce both articular and bone OA damage.

Our data demonstrated that a substance which is probably able to interfere on cartilage catabolism, to help the promotion of new cartilage synthesis and to reduce oxidative damage to the joint, like native COLLII partially hydrolyzed, could represent a further new therapeutic possibility to improve hand $\mathrm{OA}$, even in association with other SYSADOAs (Symptomatic slow acting drugs for osteoarthritis). Trentham has specified that several studies have shown significant improvement in symptoms when patients were supplemented with undenatured COLLII, including improved joint mobility and flexibility, reduced joint pain, and, in some patients, complete remission of symptoms. The fact that OA is often characterized by an underlying immune disorder lends itself to likelihood that an immune-enhancing nutrient such as undenatured COLLII could be useful in reducing inflammation and redness symptoms of OA, as well [24]. Bagchi et al. confirm the efficacy of undenatured COLLII in OA and RA in a pilot study in humans [25]. D'Altilio et al. in a placebo-controlled study demonstrated that daily treatment of arthritis-affected dogs with COLLII alone or in combination with GC markedly alleviates arthritis-associated pain and is well tolerated as no side effect was noted [26].

Moreover, evidence of the better uCTX-I correlation in the EOA subgroup, even considering the smaller case record, could suggest that EOA might have greater affinity with metabolic bone diseases, like osteoporosis [27, 28].

Further studies are necessary to confirm the efficacy of COLLII as a protective factor of OA cartilage, and the potential significance of uCTX-I as a further marker to assess the evolution of EOA bone damage.

Conflict of interest The authors declare that they have no conflict of interest related to the publication of this manuscript.

\section{References}

1. Ihyc A, Osiecka-Iwan A, Jozwiak J, Moskalewski S (2001) The morphology and selected biological properties of articular cartilage. Ortop Traumatol Rehabil 3(2):151-162

2. Morales TI (2007) Chondrocyte moves: clever strategies? Osteoarthritis Cartilage 15(8):861-871

3. Reijman M, Hazes JM, Bierma-Zeinstra SM, Koes BW, Christgau F, Christiansen C, Uitterlinden AG, Pols HA (2004) A new marker for osteoarthritis: cross-sectional and longitudinal approach. Arthritis Rheum 50(8):2471-2478

4. Charni N, Juillet F, Garnero P (2005) Urinary type II collagen helical peptide (HELIX-II) as a new biochemical marker of cartilage degradation in patients with osteoarthritis and rheumatoid arthritis. Arthritis Rheum 52(4):1081-1090

5. Jordan KM, Syddall HE, Garnero P, Gneyts E, Dennison EM, Sayer AA, Delmas PD, Cooper C, Arden NK (2006) Urinary CTX-II and glucosyl-galactosyl-pyridinoline are associated with the presence and severity of radiographic knee osteoarthritis in men. Ann Rheum Dis 65(7):871-877

6. Bruyere O, Collette J, Kothari M, Zaim S, White D, Genant H, Peterfy C, Burlet N, Ethgen D, Montague T, Dabrowski C, Reginster JY (2006) Osteoarthritis, magnetic resonance imaging, and biochemical markers: a one year prospective study. Ann Rheum Dis 65(8):1050-1054

7. Conrozier T, Ferrand F, Poole AR, Verret C, Mathieu P, Ionescu M, Vincent F, Piperno M, Spiegel A, Vignon E (2007) Differences in biomarkers of type II collagen in atrophic and hypertrophic osteoarthritis of the hip: implications for the differing pathobiologies. Osteoarthritis Cartilage 15(4):462-467

8. Sharif M, Kirwan J, Charni N, Sandell LJ, Whittles C, Garnero P (2007) A 5-yr longitudinal study of type IIA collagen synthesis and total type II collagen degradation in patients with knee osteoarthritis-association with disease progression. Rheumatology 46(6):938-943

9. Garnero P, Charni N, Juillet F, Conrozier T, Vignon E (2006) Increased urinary type II collagen helical and $\mathrm{C}$ telopeptide levels are independently associated with a rapidly destructive hip osteoarthritis. Ann Rheum Dis 65:1639-1644

10. Mazières B, Garnero P, Guéguen A, Abbal M, Berdah L, Lesquesne M, Nguyen M, Salles J-P, Vignon E, Dougados M (2006) Molecular markers of cartilage breakdown and synovitis at baseline as predictors of structural progression of hip osteoarthritis. The ECHODIAH Cohort. Ann Rheum Dis 65:354-359

11. Meulenbelt I, Kloppenburg M, Kroon HN, Houwing-Dustermaat JJ, Garnero P, Hellio Le Graverand MP, DeGroot J, Slagboom PE (2006-2007) Urinary CTX-II levels are associated with radiographic subtypes of osteoarthritis in hip, knee, hand, and facet joints in subject with familial osteoarthritis at multiple sites: the GARP study. Ann Rheum Dis 65:360-365-Osteoarthritis Cartilage 15:379-385

12. Altman R, Alarcon G, Appelrouth D, Bloch D, Borenstein D, Brandt K, Brown C, Cooke TD, Daniel W, Gray R, Greenwald R, Hochberg M, Howell D, Ike R, Kapila P, Kaplan D, Koopman W, Longley S, McShane DJ, Medsger T, Michel B, Murphy W, Osial T, Ramsey-Goldman R, Rothschild B, Stark K, Wolfe F (1990) The American College of Rheumatology criteria for the classification and reporting of osteoarthritis of the hand. Arthritis Rheum 33:1601-1610

13. Kellgren JH (1963) The epidemiology of chronic rheumatism. In: Davis FA (ed) Atlas of standard radiographs of arthritis. vol II. Blackwell Scientific Publication, Philadelphia, pp 10-1114

14. Lorenz H, Wenz W, Ivancic M, Steck E, Richter W (2005) Early and stable upregulation of collagen type II, collagen type I and YKL40 expression levels in cartilage during early experimental osteoarthritis occurs independent of joint localization and histological grading. Arthritis Res Ther 7(1):R156-R165

15. Bettica P, Cline G, Hart DJ (2002) Evidence for increased bone resorption in patients with progressive knee osteoarthritis: longitudinal results from the Chingford study. Arthritis Rheum 46(12):3178-3184

16. Sandell LJ, Ainier T (2001) Articular cartilage and changes in arthritis. An introduction: cell biology of osteoarthritis. Arthritis Res 3:107-113

17. Lajeunesse D (2007) Does subchondral bone tissue remodeling or cell function play a role in articular cartilage loss in osteoarthritis? Osteoarthritis Cartilage 15(Suppl):C3

18. Marcelli C, Favier F, Kotzki PO, Ferrazzi V, Picot MC, Simon L (1995) The relationship between osteoarthritis of the hands, bone 
mineral density, and osteoporotic fractures in elderly women. Osteoporos Int 5(5):382-388

19. Zoli A, Lizzio MM, Capuano A, Massafra U, Barini A, Ferraccioli G (2006) Osteoporosis and bone metabolism in postmenopausal women with osteoarthritis of the hand. Menopause 13(3):462-466

20. Ramsbottom H, Lockwood B (2006) Nutriceutical for healthy joints. Pharmacogenomics J 277:740-746

21. Lippiello L, Woodward J, Karpman R, Hammad TA (2000) In vivo chondroprotection and metabolic synergy of glucosamine and chondroitin sulphate. Clin Orthop Relat Res 381:229-240

22. Clegg DO, Reda DJ, Harris CL, Klein MA, O'Dell JR, Hooper MM, Bradley JD, Bingham CO, Weisman MH, Jackson CG, Lane NE, Cush JJ, Moreland LW, Schumacher HR Jr, Oddis CV, Wolfe F, Molitot JA, Yocum DE, Schnitzre TJ, Furst DE, Sawitzke AD, Shi H, Brandt KD, Moskowitz RW, Williams HJ (2006) Glucosamine, chondroitin sulphate, and the two in combination for painful knee osteoarthritis. $\mathrm{N}$ Engl $\mathrm{J}$ Med 354(8):795-808

23. Pelletier JP, Martel-Pelletier J (2007) DMOAD developments: present and future. Bull NYU Hosp Jt Dis 65(3):242-248

24. Trentham DE, Halpner AD, Trentham RA, Bagchi M, Kothari S, Preuss HG, Bagchi D (2001) Use of undenatured type II collagen in the treatment of rheumatoid arthritis. Clin Pract Altern Med 2:254-259

25. Bagchi D, Misner B, Bagchi M, Cothari SC, Downs BW, Fafard RD, Preuss HG (2002) Effects of orally administered undenatured type II collagen against arthritic inflammatory diseases: a mechanistic exploration. Int J Clin Pharmacol Res 22(3-4):101110

26. D'Altilio M, Peal A, Alvey M, Simms C, Curtsingrer A, Gupta RC, Canerdy TD, Good JT, Magchi M, Bagchi D (2007) Therapeutic efficacy and safety of undenatured type II collagen singly or in combination with glucosamine and chondroitin in arthritic dogs. Toxicol Mech Methods 17(4):189-196

27. El-Sherrif HE, Kamal R, Moawyah O (2008) Hand osteoarthritis and bone mineral density in postmenopausal women; clinical relevance to hand function, pain and disability. Osteoarthritis Cartilage 168(1):12-17

28. Sowers M, Lachance L, Jamadar D, Hochberg MC, Hollis B, Crutchfield M, Jannausch ML (1999) The associations of bone mineral density and bone turnover markers with osteoarthritis of the hand and knee in pre-and perimenopausal women. Arthritis Rheum 42(39):483-489 